\title{
Magnetospheric mapping of the dayside UV auroral oval at Saturn using simultaneous HST images, Cassini IMF data, and a global magnetic field model
}

\author{
E. S. Belenkaya ${ }^{1}$, S. W. H. Cowley ${ }^{2}$, J. D. Nichols ${ }^{2}$, M. S. Blokhina ${ }^{1}$, and V. V. Kalegaev ${ }^{1}$ \\ ${ }^{1}$ Institute of Nuclear Physics, Moscow State University, Vorob'evy Gory, 119992 Moscow, Russia \\ ${ }^{2}$ Department of Physics \& Astronomy, University of Leicester, Leicester LE1 7RH, UK \\ Received: 26 January 2011 - Revised: 22 June 2011 - Accepted: 25 June 2011 - Published: 8 July 2011
}

\begin{abstract}
We determine the field-aligned mapping of Saturn's auroras into the magnetosphere by combining UV images of the southern dayside oval obtained by the Hubble Space Telescope (HST) with a global model of the magnetospheric magnetic field. The model is tailored to simulate prevailing conditions in the interplanetary medium, corresponding to high solar wind dynamic pressure and variable interplanetary magnetic field (IMF) strength and direction determined from suitably lagged field data observed just upstream of Saturn's dayside bow shock by the Cassini spacecraft. Two out of four images obtained in February 2008 when such simultaneous data are available are examined in detail, exemplifying conditions for northward and southward IMF. The model field structure in the outer magnetosphere and tail is found to be very different in these cases. Nevertheless, the dayside UV oval is found to have a consistent location relative to the field structure in each case. The poleward boundary of the oval is located close to the open-closed field boundary and thus maps to the vicinity of the magnetopause, consistent with previous results. The equatorward boundary of the oval then maps typically near the outer boundary of the equatorial ring current appropriate to the compressed conditions prevailing. Similar results are also found for related images from the January 2004 HST data set. These new results thus show that the mapped dayside UV oval typically spans the outer magnetosphere between the outer part of the ring current and the magnetopause. It does not encompass the region of primary corotation flow breakdown within the inner Enceladus torus.
\end{abstract}

Keywords. Magnetospheric physics (Auroral phenomena; Planetary magnetospheres; Solar wind-magnetosphere interactions)

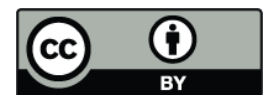

Correspondence to: E. S. Belenkaya (elena@dec1.sinp.msu.ru)

\section{Introduction}

This paper is concerned with the structure of the magnetic field in the outer regions of Saturn's magnetosphere, specifically the mapping of field lines from the high-latitude ionosphere to the equatorial regions and magnetopause, and what one can learn from this about the mapping and origins of Saturn's polar auroras. Previous work using the paraboloid model of Saturn's magnetosphere (Alexeev et al., 2006; Belenkaya et al., 2006a), also employed here, has shown that this field structure is complex, and depends on the strength and direction of the interplanetary magnetic field (IMF) (Belenkaya et al., 2006b, 2007, 2008). The field also depends on the solar wind dynamic pressure which changes the overall size of the magnetosphere via pressure balance at the magnetopause boundary (Kanani et al., 2010). This also modulates the strength of the ring current, such that the field in the middle magnetosphere is quasi-dipolar in form when the magnetosphere is compressed but extends into a magnetodisc when it is expanded (Alexeev et al., 2006; Bunce et al., 2007, 2008b; Arridge et al., 2008).

As discussed recently e.g. by Stallard et al. (2007a, b) and Kurth et al. (2009), the physical processes which result in the generation of Saturn's auroras are not yet well established. With increasing equatorial distance from the planet, field-aligned currents and auroras could firstly result from corotation breakdown in the Enceladus torus through plasma production and transport processes analogous to those at Jupiter discussed by Hill (1979) and Pontius and Hill (1982). At larger distances in the outer magnetosphere Sittler et al. (2006) have discussed the role of centrifugally-driven instabilities, while Cowley et al. (2004a, b, 2008) have proposed that corotation breakdown near the open-closed field line boundary plays an essential role. We now briefly discuss each of these in turn. 
Firstly, field-aligned currents and auroras could be associated with the corotation breakdown in the inner and middle magnetosphere beginning at radial distances of $\sim 3-4 R_{\mathrm{S}}$ (Wilson et al., 2009), which is associated with water-group ion production and outward transport in the Enceladus torus (Saur et al., 2004; Pontius and Hill, 2009). According to this picture, the plasma flow should return to near-corotation immediately equatorward of the main auroral oval. However, Stallard et al. (2007b) have presented a detailed analysis of profiles of the infrared (IR) $\mathrm{H}_{3}^{+}$emission intensity and Doppler velocity across Saturn's polar auroral region, and show that this signature is not observed in their data. These authors thus conclude that this process is not responsible for the generation of the main auroral oval at Saturn. However, they identify IR emissions at lower latitudes than the main oval which may be associated with the corotation enforcement currents in the inner and middle magnetosphere, which have been further studied by Stallard et al. (2010).

Secondly, Sittler et al. (2006) have presented a model of Saturn's global auroral response to the solar wind as observed in simultaneous Hubble Space Telescope (HST) auroral images and Cassini upstream measurements of the solar wind and IMF obtained in January 2004, emphasizing that Saturn's magnetosphere is a fast rotator. They argue that the torques on Saturn's outer magnetosphere are relatively low, such that its outer magnetosphere will tend to conserve angular momentum. When compressed on the dayside the outer magnetosphere will then spin up, and when it expands it will spin down. These authors consider the outer boundary of the plasma sheet at $L \sim 15$ (where $L$ is the equatorial radial distance in planetary radii) to be the primary source of precipitating auroral particles. They suggest that radial transport is dominated by centrifugally-driven flux tube interchange motions, such that when the magnetosphere is compressed and spins up, outward transport will increase, and the precipitating particles will move radially outward. This mechanism will thus cause the auroral oval to move to higher latitudes as observed.

Thirdly, based on Voyager plasma observations on closed field lines in the near-equatorial magnetosphere and IR Doppler observations on open field lines in the polar cap, Cowley et al. (2004a) show that a ring of upward-directed currents should flow in the vicinity of the boundary between open and closed field lines that is of sufficient intensity to require significant acceleration of magnetospheric electrons, resulting in ultra violet (UV) auroral emissions of a few tens of kilo-Rayleighs (kR). Stallard et al. (2007b) note that the characteristic signature of this model is a significant velocity shear in the region of the main oval, corresponding to the open-closed field line boundary, in which the plasma on the equatorward side should be closer to rigid corotation than that on the poleward side. Stallard et al. (2007b) suggested that the fact that this signature is not observed in their data may be explained by their spatial resolution, which did not enable them to resolve a possible narrow band of enhanced rotation equatorward of the main auroral oval. Initial evidence in favour of this model has been presented by Bunce et al. (2008a), who analysed near-simultaneous observations of Saturn's southern auroras using HST UV images and concurrent Cassini measurements of auroral field-aligned currents in the polar magnetosphere. The Cassini data provide evidence of strong upward currents flowing in the open-closed boundary region at noon, co-located with the main oval observed in the HST images.

Belenkaya et al. (2006b, 2007, 2008, 2010) have studied the relationship between Saturn's aurora and the IMFdependent field structure of the outer magnetosphere using the paraboloid field model, specifically examining the correspondence between the auroral oval and the boundary between open and closed field lines. Such studies require the simultaneous availability of both UV auroral images obtained by the HST and IMF data upstream from Saturn obtained by Cassini, of which only two such joint campaign data sets exist at present. Belenkaya et al. (2006b, 2007, 2008) studied the joint data obtained during Cassini approach to the planet in January 2004 when the spacecraft lay at radial distances of $\sim 1300 R_{\mathrm{S}}$, such that the spacecraft-planet IMF propagation time was $\sim 20 \mathrm{~h}$ with uncertainties of several hours. $\left(R_{\mathrm{S}}\right.$ is Saturn's 1 bar equatorial radius equal to $60268 \mathrm{~km}$.) Subsequently, Belenkaya et al. (2010) studied HST auroral images obtained on an approximately daily basis during a twoweek period in February 2008 (see Clarke et al., 2009, for further campaign details). For much of this interval Cassini was located inside Saturn's magnetosphere, but it emerged into the solar wind near apoapsis on the dayside of the planet during DOY (day of year) 43 to 46 , such that the upstream IMF could then be monitored with much reduced propagation delay and uncertainty. Belenkaya et al. (2010) used the paraboloid magnetosphere model, including the fields of the ring current, tail current, and magnetopause current, together with a partially-penetrated IMF, to calculate the boundary of the open field line region in the southern ionosphere, it being noted that only the southern aurora could be observed during both campaign intervals due to the pre-equinox conditions prevailing (vernal equinox occurred at Saturn in $\mathrm{Au}-$ gust 2009). Overall, the results from both the 2004 and 2008 data intervals show a close relationship, with the openclosed boundary lying close to the poleward boundary of the auroras.

In this paper we further analyse and discuss the modelling results obtained using the joint HST-Cassini data from February 2008. Specifically, we elucidate the structure of the magnetospheric magnetic field pertaining to the auroral imaging intervals using the paraboloid model, thus allowing us to understand the connection between the southern polar ionosphere and domains within the equatorial magnetosphere and/or magnetopause. We then map the model field lines from the poleward and equatorward boundaries of the observed dayside UV oval into the magnetosphere in order to understand the physical domains concerned, thus helping to 


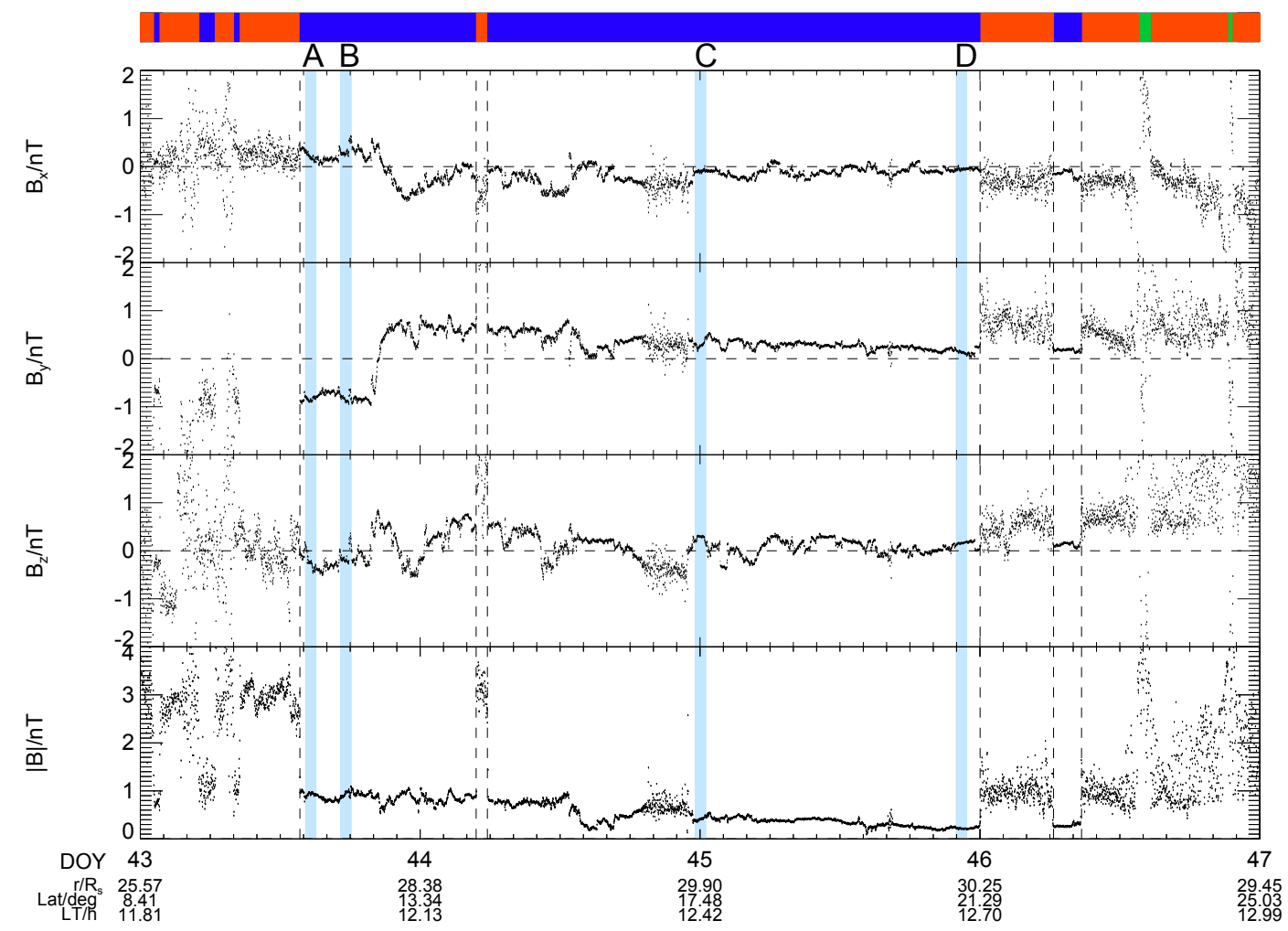

Fig. 1. Cassini magnetic field data for DOY 43 to 46 of 2008. From top to bottom the data panels show the three components of the magnetic field in KSM coordinates, and the field magnitude. In the colour-coded region identifier at the top of the plot green corresponds to the magnetosphere, red to the magnetosheath, and blue to the solar wind. Principal bow shock crossings (red-blue transitions) are identified by vertical black dashed lines. The data at the bottom of the plot give the radial distance $\left(R_{\mathrm{S}}\right)$, latitude $(\mathrm{deg})$, and local time (h) of the spacecraft. The $1 \mathrm{~h}$ blue vertical stripes labelled "A" to " $\mathrm{D}$ " correspond to the suitably lagged times of four HST imaging intervals. (From Belenkaya et al., 2010).

clarify the relationship between the observed emissions and the suggested auroral mechanisms discussed above. We also briefly compare our results with those derived from selected related images from the January 2004 data set.

\section{Cassini and HST observations during DOY 43-46 2008}

In this section we first discuss the Cassini data and HST images which form the basis of the analysis presented here, taken from the results of Belenkaya et al. (2010). In Fig. 1 we show magnetic field data obtained by the Cassini fluxgate magnetometer (Dougherty et al., 2004) for DOY 43 to 46 of 2008. From top to bottom we show the three components of the magnetic field in kronocentric solar-magnetospheric (KSM) coordinates, together with the field magnitude. In the KSM system X points towards the Sun (approximately anti-parallel to the solar wind flow), the X-Z plane contains the planet's magnetic (and spin) axis, and $\mathrm{Y}$ completes the right-hand orthogonal triad pointing towards dusk. Spacecraft position information is given at the bottom of the fig- ure, specifically the radial distance $\left(R_{\mathrm{S}}\right)$, latitude (deg), and LT (h). A colour-coded region identifier is shown at the top of the figure, where blue corresponds to the solar wind, red to the magnetosheath, and green to the magnetosphere. Principal bow shock crossings (red-blue transitions) are identified by vertical black dashed lines. It can be seen that the spacecraft was consistently located in the solar wind just upstream of Saturn's dayside bow shock, at $\sim 12: 00 \mathrm{~h} \mathrm{LT}$ and $\sim 10^{\circ}-20^{\circ}$ latitude, from the middle of DOY 43 to the end of DOY 45 , except for a short interval in the first half of DOY 44 as marked. It can be seen that the IMF strength in the solar wind declined from $\sim 1 \mathrm{nT}$ near the beginning of the interval to $\sim 0.25 \mathrm{nT}$ towards its end.

Belenkaya et al. (2010) estimated that for this interval of data, the configuration of the ionospheric field and flow responds to solar wind conditions with an overall delay of about $6 \mathrm{~h}$. This, together with the $\sim 1 \mathrm{~h}$ light propagation time to Earth, was thus adopted to determine the Cassini solar wind interval corresponding to particular HST images. Four such images were obtained during the interval, labelled "A" to "D", each consisting of the sum of nine consecutive $100 \mathrm{~s}$ images of Saturn's southern oval obtained using 

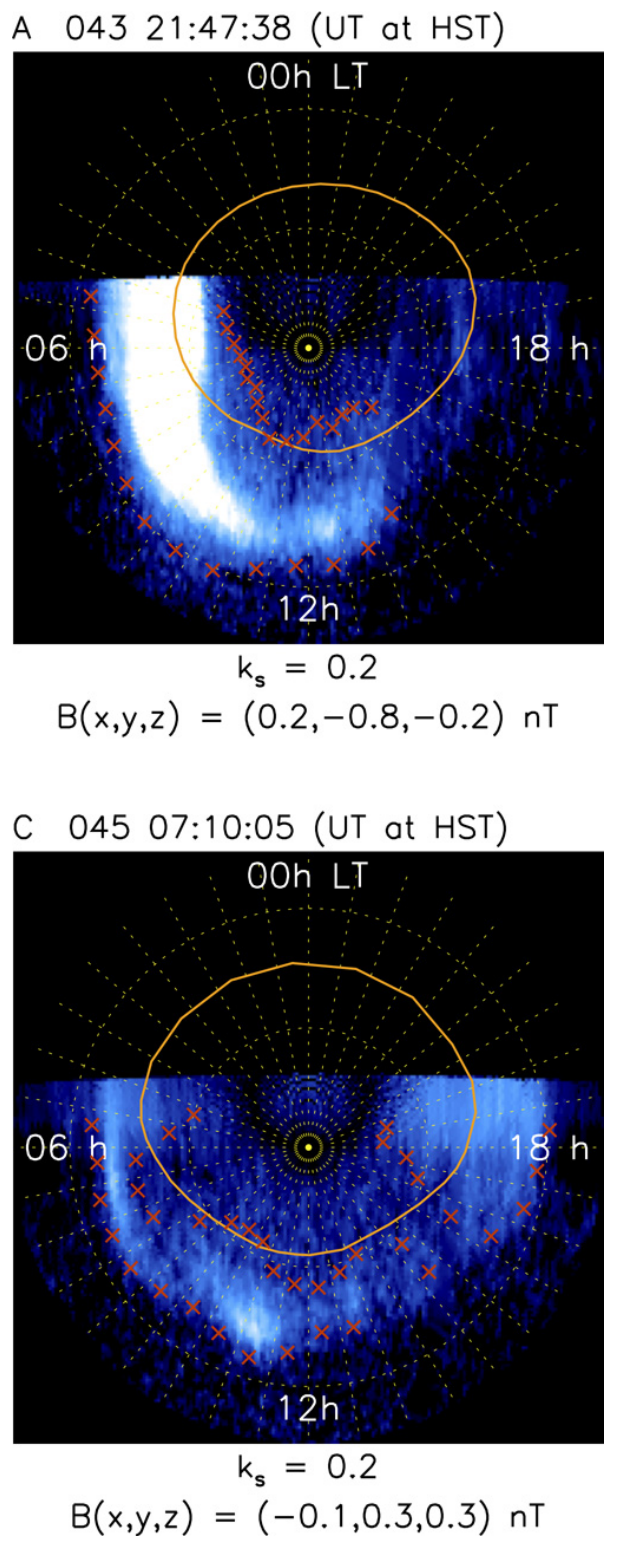

Fig. 2. Plots showing two HST UV images of Saturn's southern auroras, projected onto a spheroidal surface $1100 \mathrm{~km}$ above the atmospheric 1 bar level, with noon at the bottom and dawn to the left. The view is thus "through" the planet from the north. Dotted yellow lines show a latitude-longitude grid at $10^{\circ}$ intervals in each case. Only the dayside portion of the oval was well-observed due to the pre-equinoctial conditions prevailing, such that the images are truncated somewhat beyond the dawn-dusk meridian. Image identifiers are given at the top of each panel together with the start time of the $\sim 20$ min combined exposure time. The locations of the poleward and equatorward boundaries of the emission in each image are shown by red crosses, determined at $10^{\circ}$ intervals of longitude from a $5 \mathrm{kR}$ limiting emission intensity. The superposed solid orange lines show the open field regions for $k_{\mathrm{S}}=0.2$ in each case, calculated using the paraboloid magnetic field model and the corresponding averaged IMF vector determined from Cassini data, indicated in rounded form at the bottom of each panel. the F115LP long-pass filter, combined together to increase signal-to-noise. The blue vertical stripes in Fig. 1 thus show the solar wind times corresponding to these images, labelled A to D at the top of the figure, each stripe showing the $1 \mathrm{~h}$ interval over which the IMF data were averaged in order to obtain a representative value. It can be seen in particular that the north-south field component $B_{Z}$ was negative (southward) during images $\mathrm{A}$ and $\mathrm{B}$, but was positive (northward) during images $C$ and $D$. In this paper we focus on images $A$ and $\mathrm{C}$ as representative of these two conditions.

The images are presented in Fig. 2, projected onto a southern latitude and longitude grid shown by the yellow dotted lines at intervals of $10^{\circ}$ each. The view is "through" the planet from the north with noon at the bottom of each plot and dawn to the left. Because the sub-Earth latitude of Saturn was $-8^{\circ}$ at the time of these observations, only the dayside portion of the oval was well-observed, such that the images are truncated somewhat beyond the dawn-dusk meridian. The red crosses in Fig. 2 indicate the poleward and equatorward boundaries of the auroral emission, used below to map the auroral boundaries along field lines into the magnetosphere. These were determined by averaging the emission over $10^{\circ}$ longitude bins to increase the signal to noise, and applying a simple emission threshold of $5 \mathrm{kR}$. This value was set sufficiently high that the boundary locations are not greatly affected by noise in the data, and sufficiently low that significant auroral emission is not omitted. We also eliminate a few points whose co-latitude lies more than two standard deviations from the mean in each image, which occur in regions of weak emission where a clear visual boundary cannot be identified. Even so, the poleward boundary of the auroras is notably variable in these images, while the equatorward boundary is relatively unvarying. We note that no corrections have been made to the boundaries for artificial poleward stretching due to the finite height of the auroral curtain, since this effect is small compared with the significant difference in morphology between the images. It can be seen that the UV oval in image $\mathrm{A}$ is brightest in the dawn-to-noon sector, extending between $\sim 6^{\circ}$ and $\sim 18^{\circ}$ southern co-latitude, while being more uniformly distributed in image $\mathrm{C}$, extending between $\sim 10^{\circ}$ and $\sim 16^{\circ}$. These values are comparable with the co-latitudes of the boundaries of the southern UV dayside oval determined from a wider set of UV images by Badman et al. (2006), who found that the median poleward boundary lies at $14^{\circ}$ and the median equatorward boundary at $16^{\circ}$, both within considerable variability. We thus note that these auroral distributions are entirely representative of a much wider body of such data (e.g., Clarke et al., 2009).

The data at the bottom of each image in Fig. 2 give the corresponding rounded averaged IMF vector determined from the Cassini data in Fig. 1. As we go on in the next section to describe, the (unrounded) averaged IMF vectors have been employed in the paraboloid model of Saturn's magnetosphere to calculate the position of the open-closed field line boundary, shown by the orange lines in each image. Those 
shown here represent a higher-resolution refinement of those previously depicted by Belenkaya et al. (2010).

\section{Paraboloid model calculations}

In the paraboloid model (Alexeev et al., 2006; Belenkaya et al., 2006a, 2007, 2008, 2010; Alexeev, 2010), the magnetopause is taken to be a paraboloid of revolution about the Saturn-Sun line, such that the model is expressed in KSM coordinates. The main contributors to the model magnetic field are (i) the intrinsic magnetic (dipole) field of the planet, together with the shielding magnetopause current which confines the dipole field inside the boundary, (ii) the ring current and the corresponding shielding magnetopause current, (iii) the tail currents and their closure currents on the magnetopause, and (iv) the IMF which partially penetrates into the magnetosphere. The parameters which define the model magnetic field are then as follows. (i) $\Psi$ is the tilt angle between the magnetic dipole axis and the KSM Z-axis ( $-8.4^{\circ}$ during DOY $43-462008$ ), (ii) $R_{\mathrm{SS}}$ is the distance from Saturn's centre to the subsolar point on the magnetopause, (iii) $R_{\mathrm{rc} 1}$ and $R_{\mathrm{rc} 2}$ are the distances to the outer and inner edges of the ring current, respectively, (iv) $B_{\text {rc1 }}$ is the radial component of the ring current field at the outer edge of the ring current, (v) $R_{2}$ is the distance from the planet's centre to the inner edge of the magnetospheric tail current sheet, and (vi) the field magnitude of the tail currents at the inner edge of the tail current sheet is $B_{\mathrm{t}} / \alpha_{0}$, where $\alpha_{0}=\left(1+2 R_{2} / R_{\mathrm{ss}}\right)^{1 / 2}$. For simplicity the current sheets in the model are taken to be of zero thickness, compared with observed thicknesses typically of a few $R_{\mathrm{S}}$ (e.g., Kellett et al., 2009), which should have little effect on the field line mapping between ionosphere and magnetosphere. Further discussion of the paraboloid model current systems and magnetic fields may be found, e.g., in Alexeev and Belenkaya (2005). The effect of the IMF inside the magnetosphere is given by adding the uniform field $k_{\mathrm{S}} \boldsymbol{B}_{\mathrm{IMF}}$, where $\boldsymbol{B}_{\mathrm{IMF}}$ is the IMF vector and $0 \leq k_{\mathrm{S}} \leq 1$ is the magnetosphere penetration coefficient. We note that the model is steadystate and thus does not include representations of the global magnetic field perturbations that rotate around the planet near the planetary period (e.g., Andrews et al., 2010), related to corresponding small-amplitude "wobbles" of the auroral oval (e.g., Nichols et al., 2010), nor of the transient plasmoids possibly associated with the Vasyliunas-cycle observed in Saturn's nightside plasma sheet (e.g., Jackman et al., 2008). The model also does not include representation of the quasisteady magnetosphere-ionosphere currents that couple angular momentum between these two regions (e.g., Hill, 1979; Cowley et al., 2004a), that generally lead to "lagging" field lines in the magnetosphere in the presence of sub-corotation. No detailed empirically-based models of such fields have yet been derived for Saturn due to the dominant presence of the above "planetary period" oscillations, however, simple esti- mates indicate modest LT displacements that are largely directed around the auroral oval, thus not significantly affecting the radial mapping issue.

In the field calculations shown here we keep the basic model parameters fixed as in Belenkaya et al. (2010), so that we can clearly discern the effect of the variable IMF. Since according to the results presented by Clarke et al. (2009) the interval studied corresponds overall to one in which Saturn's magnetosphere was quite strongly compressed by the solar wind, with the dynamic pressure peaking at $\sim 0.1 \mathrm{nPa}$ on DOY 44 and 45 , here we choose to use a relatively compressed model throughout. Specifically we employ the model derived by Belenkaya et al. (2006a) from fits to the magnetic field observed during the Pioneer-11 flyby, which corresponds to a dynamic pressure of $\sim 0.08 \mathrm{nPa}$. This model was also successfully employed by Belenkaya et al. (2007) to describe Saturn's magnetosphere under high solar wind dynamic pressure conditions. The corresponding set of model parameters is $R_{\mathrm{SS}}=17.5 R_{\mathrm{S}}, R_{\mathrm{rc} 1}=12.5 R_{\mathrm{S}}, R_{\mathrm{rc} 2}=6.5 R_{\mathrm{S}}$, $B_{\mathrm{rc} 1}=3.62 \mathrm{nT}, R_{2}=14 R_{\mathrm{S}}$, and $B_{\mathrm{t}}=8.7 \mathrm{nT}$. We note that the values for the ring current boundaries are in reasonable accord with the Cassini field modelling results of Bunce et al. (2007) corresponding to similar compressed magnetosphere conditions.

The value of the IMF penetration coefficient $k_{\mathrm{S}}$ is not yet well known for Saturn, such that Belenkaya et al. (2010) presented results for both low $\left(k_{\mathrm{S}}=0.2\right)$ and high $\left(k_{\mathrm{S}}=0.8\right)$ values. While the structure of the outer magnetosphere and the length of the magnetospheric tail is found to be strongly affected by this choice (see their Fig. 4), the size and position of the open field region projected to the ionosphere is found to be changed only to a relatively modest degree. We also note that determinations of the IMF penetration parameter at Earth, obtained both directly using magnetospheric magnetic field data and from estimates of the "efficiency of reconnection" factor based on radar convection measurements, favour the lower value (e.g., Cowley and Hughes, 1983; Tsurutani et al., 1984; Milan et al., 2004). Here we therefore employ $k_{\mathrm{S}}=0.2$. We start by discussing the simpler situation that occurs for northward IMF corresponding to case $\mathrm{C}$.

\section{Image $C$ during northward IMF conditions}

Image $\mathrm{C}$ in Fig. 2 was obtained by the HST at $\sim 07: 00 \mathrm{UT}$ on DOY 45, with a corresponding lagged KSM IMF vector of $(-0.11,0.28,0.25) \mathrm{nT}$ such that the Z-component was northward-directed in this case (Belenkaya et al., 2010). To gain a better understanding of the magnetospheric connection to the southern polar region during this interval, we first examine the model magnetic field lines whose ionospheric footprints lie at fixed southern latitudes, at steps of $1 \mathrm{~h}$ of LT. In Fig. 3 we show these field lines for initial latitudes from $-70^{\circ}$ to $-90^{\circ}$ in steps of $4^{\circ}$, where blue and red symbols on the field lines show intersections with the planet's equatorial 

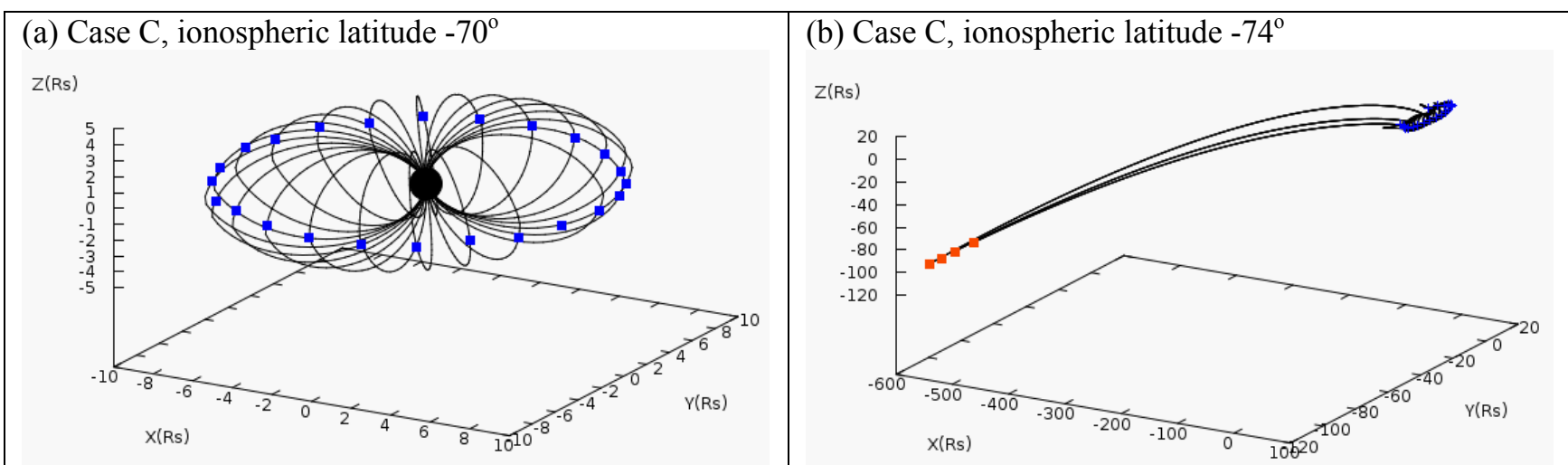

(c) Case $\mathrm{C}$, ionospheric latitude $-78^{\circ}$

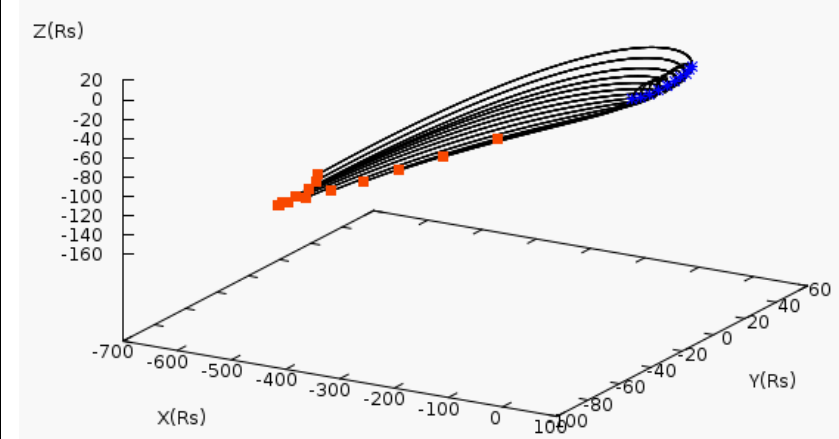

(d) Case $\mathrm{C}$, ionospheric latitude $-82^{\circ}$

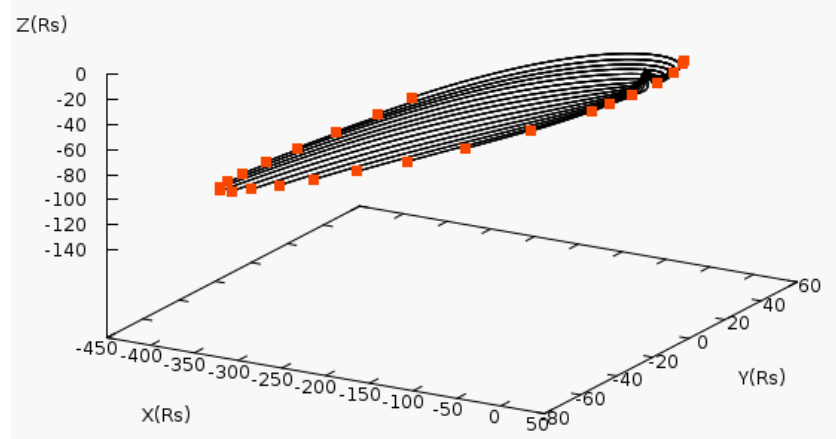

(e) Case $\mathrm{C}$, ionospheric latitude $-86^{\circ}$

(f) Case $\mathrm{C}$, ionospheric latitude $-90^{\circ}$
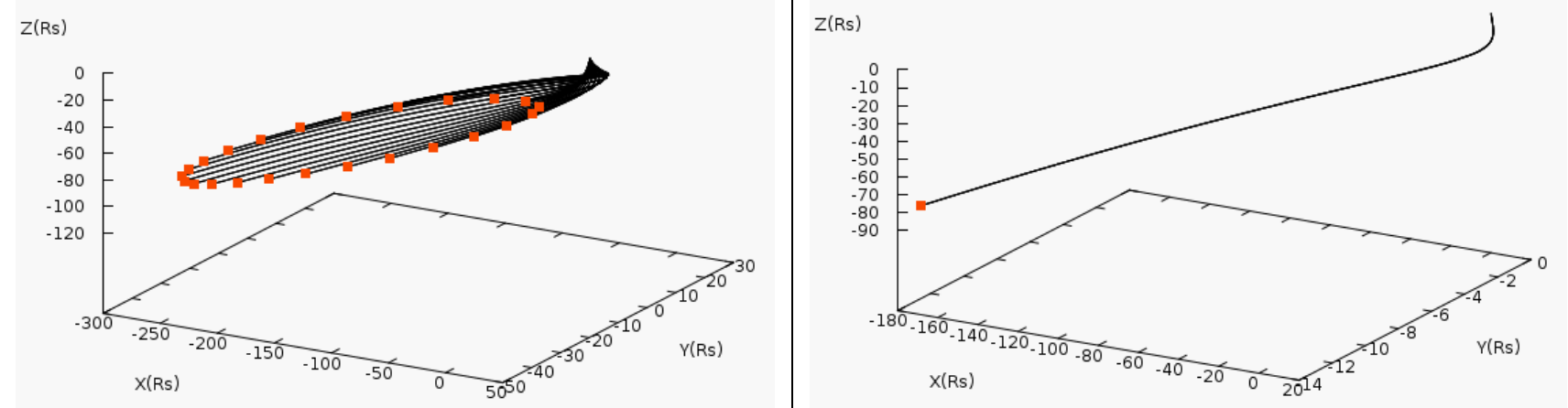

Fig. 3. Plots showing model magnetic field lines whose ionospheric footprints lie at constant southern latitudes, for the field model corresponding to case $\mathrm{C}$ with IMF penetration coefficient $k_{\mathrm{S}}=0.2$. Panels (a) to (f) show results for latitudes from $-70^{\circ}$ to $-90^{\circ}$, respectively, at steps of $4^{\circ}$. The longitude step between each field line corresponds to $1 \mathrm{~h}$ of LT. Note the varying X-Y spatial scales in the various panels. Blue symbols mark intersections of the field lines with the planet's equatorial plane, while red symbols mark intersections with the southern magnetopause. The solid black circle shows the position of Saturn.

plane and the southern magnetopause, respectively. For a footprint latitude of $-70^{\circ}$ in panel (a) it can be seen that the field lines are dipole-like, extending to radial distances of $\sim 9 R_{\mathrm{S}}$ in the equatorial plane. However, lines with footprint latitudes of $-74^{\circ}$ in panel (b) form not only closed field lines (note the change in X scale compared with panels a and b), but also open field lines stretching out into the tail (see the relevant open-closed boundary for image $\mathrm{C}$ in Fig. 2). With further increase of the footprint latitude to $-78^{\circ}$ in panel (c) the number of open field lines increases, until near $-82^{\circ}$ in panel (d), and beyond, all the field lines are open. Under such conditions of northward IMF, all the open field lines map directly from the southern ionosphere to the southern magnetopause. This is demonstrated in Fig. 4, where we show the intersections of the open field lines in Fig. 3 with the southern model magnetopause (the black dot marks the position of Saturn in the plot). The solid black lines in the figure correspond to fixed latitudes in the southern ionosphere with steps of $4^{\circ}$ from $-74^{\circ}$ to $-90^{\circ}$ as marked. The black dashed lines then correspond to fixed ionospheric LTs with steps of $1 \mathrm{~h}$, 


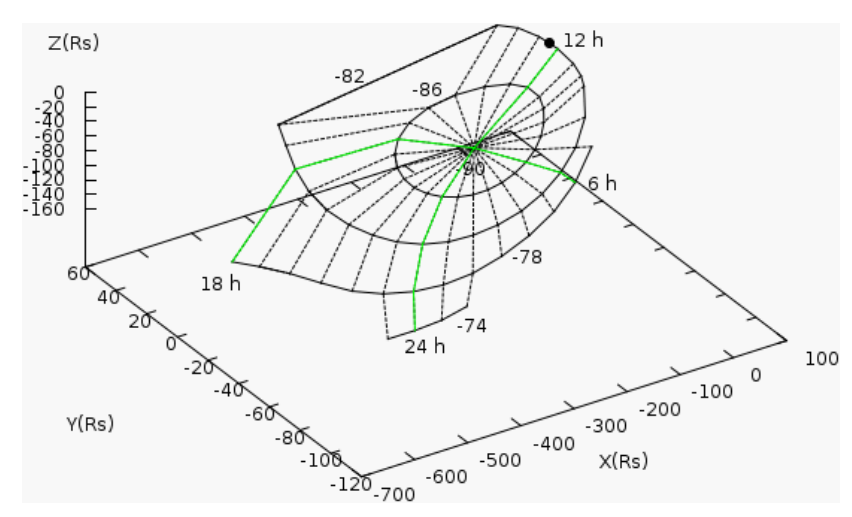

Fig. 4. Intersection points of the open magnetic field lines emerging from Saturn's southern ionosphere, as shown in Fig. 3, with the southern magnetopause, for the case of image $\mathrm{C}$ with $k_{\mathrm{S}}=0.2$. Solid curves show projections along the field of lines of constant ionospheric latitude from $-74^{\circ}$ to $-90^{\circ}$ at steps of $4^{\circ}$, while dashed lines show projections of lines of constant LT at steps of $1 \mathrm{~h}$, where the green lines show the projections of the principal meridians as marked. The black dot marks the position in the plot of Saturn.

with the principal meridians (noon, dusk, etc.) being shown by the green lines. The field line intersections are confined to the Southern Hemisphere, and also favour the dawn side of the tail due to the positive IMF Y component conditions prevailing.

We now turn specifically to the mapping of the boundaries of the southern auroras, as defined by the red crosses in the auroral image in Fig. 2. The corresponding field lines are shown in Fig. 5 in a similar format to Fig. 3, noting, however, that the ionospheric footprints are confined mainly to the dayside sector due to the limited viewing of the auroral oval by the HST. In panel (a) we show the field lines mapping from the equatorward boundary of the dayside southern oval (blue lines), consisting wholly of closed field lines in conformity with Fig. 2. These map to equatorial distances of $\sim 11.5 R_{\mathrm{S}}$ in the dawn to post-noon sector, corresponding to latitudes $\sim-73^{\circ}$ in the southern ionosphere as can be seen in Fig. 2, moving inwards to $\sim 9 R_{\mathrm{S}}$ in the dusk sector as the boundary moves equatorward to $\sim-70^{\circ}$. Panel (b) similarly shows field lines mapping from the poleward boundary of the dayside oval, some of which are closed (purple lines) while others are open (black lines), as also seen in Fig. 2. The closed lines map typically to radial distances of $\sim 16 R_{\mathrm{S}}$ near to the dayside magnetopause, while the open field lines pass into the magnetic tail in the Southern Hemisphere.

For better clarity, in Fig. 6 we show the mappings of the closed dayside oval boundary field lines in the KSM equatorial plane, together with principal features of the magnetic model. The latter features are indicated by the black solid lines, where the outer curve shows the paraboloid magnetopause, the two inner circles centred on the planet (black dot) show the inner and outer boundaries of the equatorial ring current, while the black curve across the tail on the

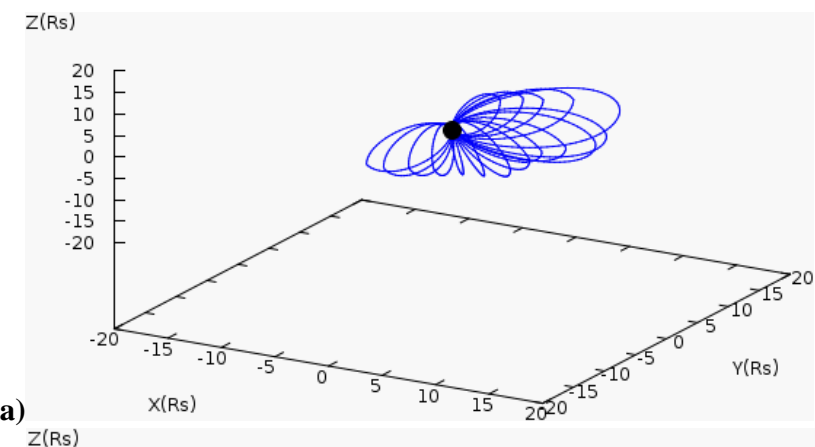

(a) $z(\mathrm{Rs})$

(b)

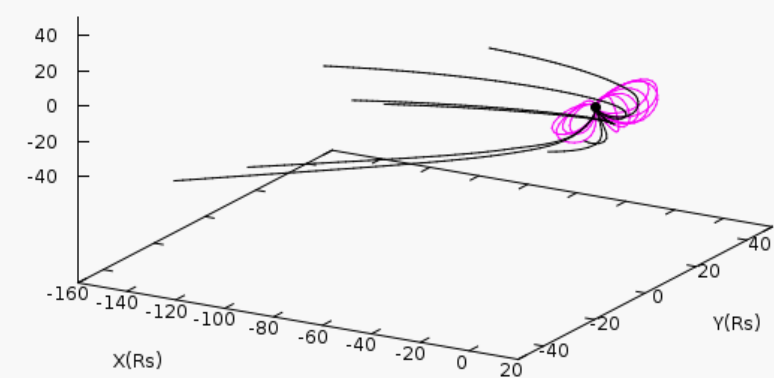

Fig. 5. Field lines mapping to (a) the equatorward and (b) the poleward boundary of the dayside UV auroral oval for image $\mathrm{C}$ with $k_{\mathrm{S}}=0.2$, corresponding in the southern ionosphere to the positions indicated by the red crosses in Fig. 2. All the field lines in the model are closed (blue lines) for the equatorward boundary in panel (a). For the poleward boundary in panel (b), some of the field lines are closed (purple) and some are open (black).

nightside indicates the inner edge of the tail current sheet. The light blue lines then show the field lines corresponding to fixed southern ionospheric latitudes of $-70^{\circ},-74^{\circ}$, and $-78^{\circ}$ as marked, while the red line corresponds to the boundary between open and closed field lines. The light blue line corresponding to $-70^{\circ}$ lies just outside the inner edge of the model ring current at $6.5 R_{\mathrm{S}}$, the latter mapping to $\sim-66^{\circ}$ in the southern ionosphere, while the line corresponding to $-74^{\circ}$ lies close to the outer edge of the model ring current at $12.5 R_{\mathrm{S}}$ on the dayside, but extends further outward to the boundary of closed field lines in the tail on the nightside.

The mapped field lines corresponding to the equatorward and poleward boundaries of the auroras are then marked by blue and purple squares in Fig. 6, respectively, all of which correspond to closed field lines since the open field lines of the poleward boundary do not intersect the equatorial plane. It can be seen that, overall, the dayside auroral oval maps from close to the dayside magnetopause at $\sim 17.5 R_{\mathrm{S}}$ for these compressed conditions (i.e. close to the boundary between open and closed field lines), to close to the outer edge of the ring current at $\sim 12 R_{\mathrm{S}}$. A modest exception occurs in our results in the dusk sector, where the equatorward boundary of the auroras moves inward toward $\sim 9 R_{\mathrm{S}}$ corresponding to the central ring current, as the latitude of the equatorward boundary in image $\mathrm{C}$ moves to $\sim-70^{\circ}$ in 


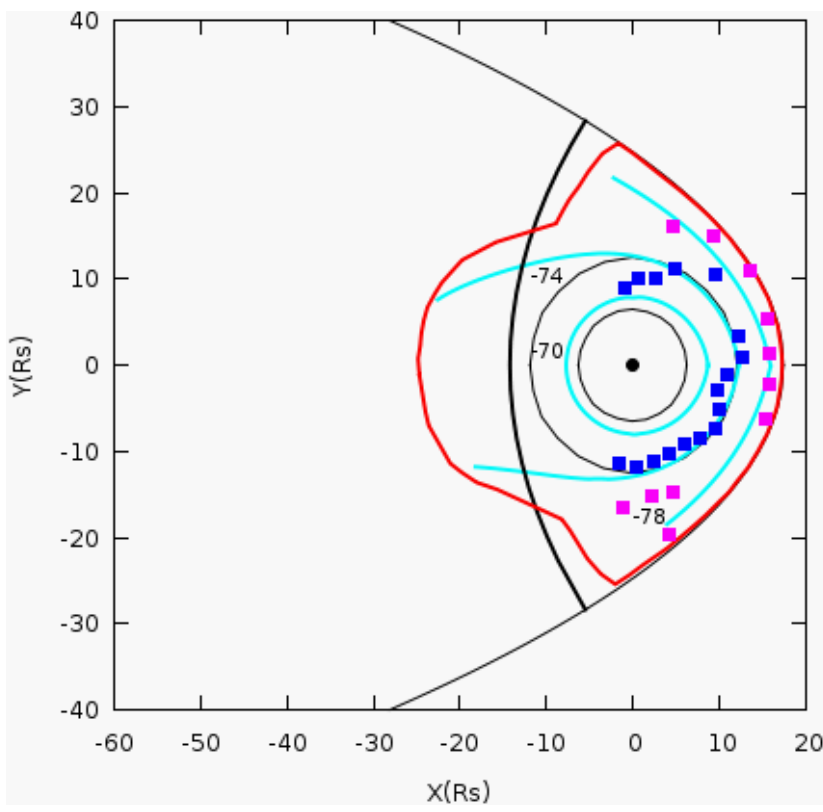

Fig. 6. Mappings of field lines into the KSM equatorial plane for image $\mathrm{C}$ with $k_{\mathrm{S}}=0.2$. The boundaries of the dayside auroral oval corresponding to the red crosses shown in Fig. 2 are shown by the blue and purple squares for the equatorward and poleward boundaries, respectively. The black lines show features of the magnetic model, where the outer curve shows the paraboloid magnetopause, the two inner circles centred on the planet (black dot) show the inner and outer boundaries of the equatorial ring current, while the black curve across the tail on the nightside indicates the inner edge of the tail current sheet. The pale blue lines show field lines corresponding to fixed southern ionospheric latitudes of $-70^{\circ},-74^{\circ}$, and $-78^{\circ}$ as marked, while the red line corresponds to the boundary between open and closed field lines.

this sector. We note that these results are in excellent accord with the findings of Talboys et al. (2009) concerning the location of the dayside upward-directed magnetosphereionosphere coupling field-aligned currents at Saturn, specifically in the southern pre-noon sector in that case. They found that the region of upward current, which may be associated with downward electron acceleration and UV auroras, maps to the closed field region between the boundary of open field lines and the outer edge of the ring current. We also note that the plasma in this outer region is dominated by hot (several $\mathrm{keV}$ and above) tenuous $\left(\sim 0.1 \mathrm{~cm}^{-3}\right)$ ions and electrons, rather than the cool $(\sim 100 \mathrm{eV}$ ions and few $\mathrm{eV}$ electrons) dense (up to $\sim 100 \mathrm{~cm}^{-3}$ ) water plasma originating from Enceladus that is characteristic of the inner region (Schippers et al., 2008; Sergis et al., 2009; Kellett et al., 2010, 2011).

As a test of the generality of these features, we have compared them with results from one of the images in the 2004 data set previously studied by Belenkaya et al. $(2007,2008)$, obtained under related interplanetary conditions. Specifi- cally, we have considered image " $m$ " in this data set, obtained on 30 January 2004 when the IMF mapped from Cassini was also northward-directed, but with a somewhat larger total magnitude of $\sim 1 \mathrm{nT}$ compared with $\sim 0.4 \mathrm{nT}$ for image $\mathrm{C}$. The compression of the magnetosphere was somewhat reduced, however, corresponding to a more usual solar wind dynamic pressure of $\sim 0.03 \mathrm{nPa}$. Differing paraboloid model parameters have thus been employed appropriate to this case (see Belenkaya et al., 2008, for details), in particular a larger subsolar magnetopause radius of $22 R_{\mathrm{S}}$. Belenkaya et al. (2008) again showed that the poleward boundary of the oval in this case maps close to the open-closed field boundary, while here we have newly computed the equatorial mapping of the equatorward boundary of the oval. As in Fig. 6 for image $\mathrm{C}$, we find that this boundary maps close to the outer edge of the model ring current in the equatorial plane, now located at a radial distance of $\sim 15 R_{\mathrm{S}}$ in this somewhat more expanded case. These results are therefore entirely compatible with those found above for image $\mathrm{C}$.

As noted previously by Belenkaya et al. (2007, 2008, 2010), the result that the poleward boundary of the UV oval lies close to the boundary of open field lines is in agreement with the model calculations of Cowley and Bunce (2003) and Cowley et al. (2004a, b, 2008), that associates the oval with upward field-aligned currents generated by a shear in azimuthal flow between open and closed field lines. Sittler et al. (2006), on the other hand, consider the outer boundary of the plasma sheet (at $L \sim 15$ in their model and at 12.5 in ours) to be the primary source of auroral precipitating particles. Our results show that generally the equatorward auroral boundary maps close to the outer edge of the ring current, such that this mechanism could also contribute to the observed emissions. Wilson et al. (2009) show that the primary corotation breakdown at Saturn occurs in the Enceladus torus between 3 and $4 R_{\mathrm{S}}$, mapping to about $-62^{\circ}$ in the southern ionosphere. This breakdown thus occurs well equatorward of the UV oval, as originally suggested by Cowley and Bunce (2003). However, corotation-enforcement currents will certainly flow in this vicinity, as discussed in the jovian context by Hill (1979), Pontius and Hill (1982) and Vasyliunas (1983). Stallard et al. (2008) reported the discovery of a lower-latitude auroral oval in IR emission at Saturn which they called a "secondary oval", lying equatorward of the main UV oval investigated here. They showed that this low-latitude emission occurs close to the point where corotation breaks down in the IR Doppler data, thus mapping to the Enceladus torus region well equatorward of the main oval (Stallard et al., 2010). Thus it seems likely that auroral emission formed by current systems caused by rigid corotation breakdown in the inner and middle magnetosphere may be realized in the lower-latitude auroral oval in IR emission, but not in the main oval. 


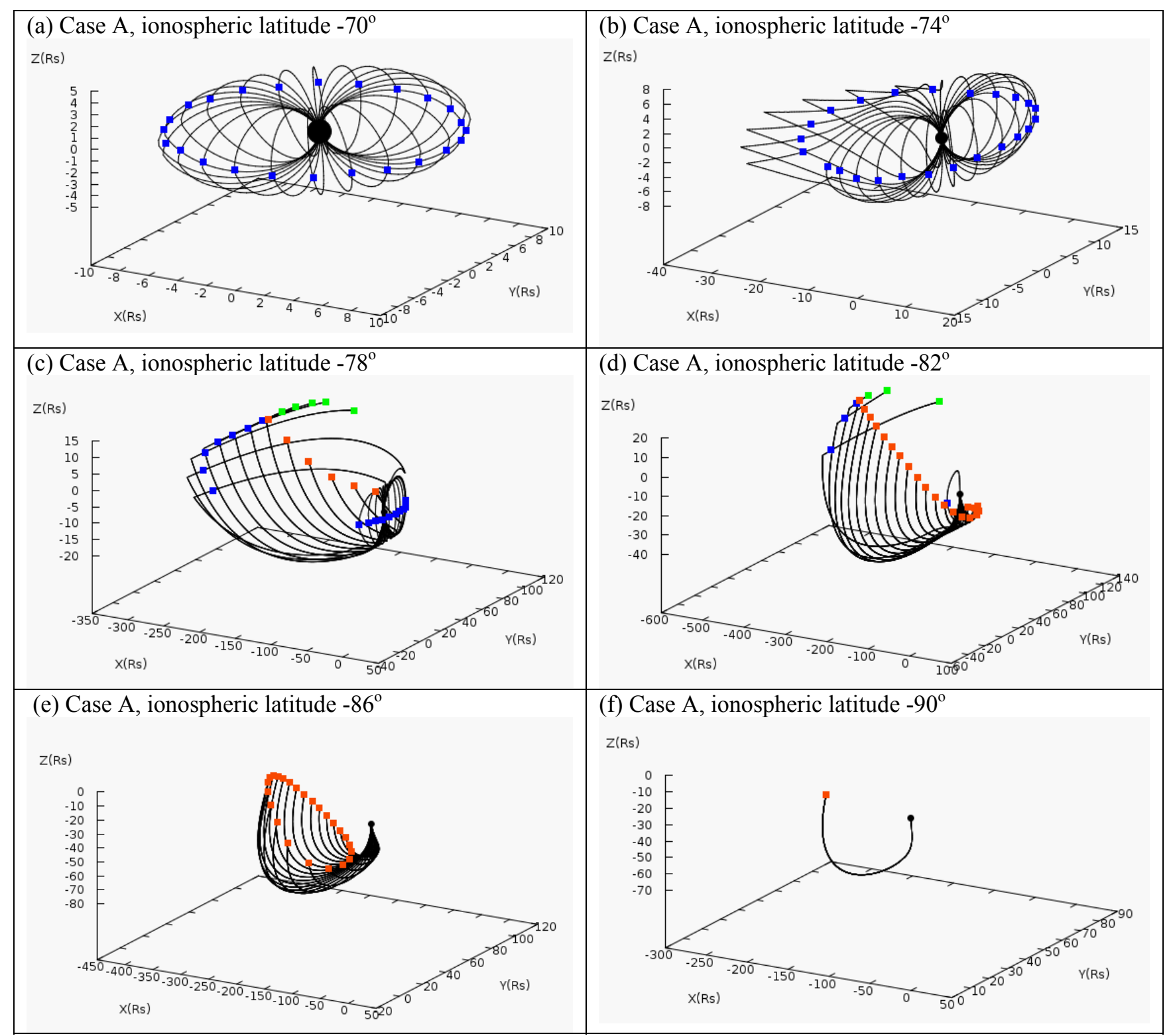

Fig. 7. Plots showing model magnetic field lines whose ionospheric footprints lie at constant southern latitudes, for the field model corresponding to case A with IMF penetration coefficient $k_{\mathrm{S}}=0.2$. The format is similar to Fig. 3 . Blue squares mark intersections of the field lines with the planet's equatorial plane, while red and green squares mark intersections with the southern and northern magnetopause, respectively.

\section{Image A during southward IMF conditions}

We now provide a similar discussion of image A in Fig. 2, obtained by the HST at $\sim 22: 00 \mathrm{UT}$ on DOY 43 with a corresponding lagged KSM IMF vector of $(0.20,-0.85,-0.24) \mathrm{nT}$, such that the Z-component was southward-directed in this case, opposite to case $\mathrm{C}$ (Belenkaya et al., 2010). Model magnetic field lines with fixed latitudes in the southern ionosphere are shown in Fig. 7 in a similar format to Fig. 3, again using an IMF penetration coef- ficient of $k_{\mathrm{S}}=0.2$. Here the blue squares again show the intersection of the field lines with the planet's equatorial plane, while the red and green squares show intersections with the southern and northern magnetopause, respectively. For an initial latitude of $-70^{\circ}$ in panel (a) it can be seen that the field lines are again dipole-like, extending to $\sim 9 R_{\mathrm{S}}$ in the equatorial plane. For $-74^{\circ}$ in panel (b) they now remain closed at all LTs, as is evident from Fig. 2, but are more stretched out into the tail on the nightside. At $-78^{\circ}$ in panel (c) open field lines appear in the dusk to midnight sector, increasing 


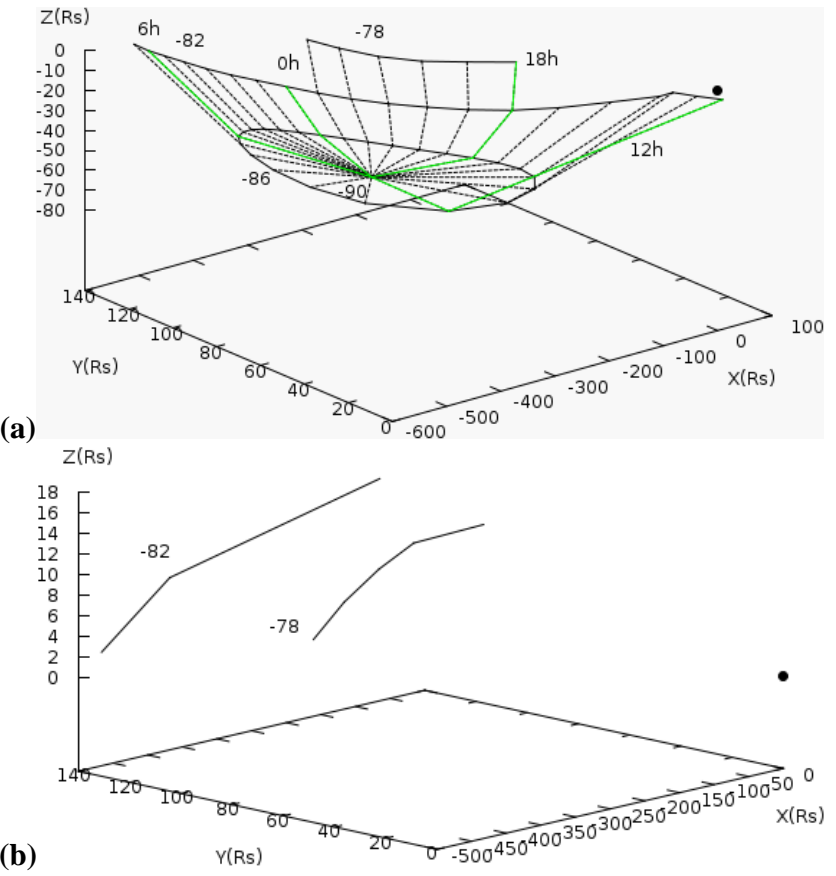

Fig. 8. Intersection points of the open magnetic field lines emerging from Saturn's southern ionosphere, as shown in Fig. 7, with (a) the southern and (b) the northern magnetopause, for the case of image A with $k_{\mathrm{s}}=0.2$. The format is the same as Fig. 4 .

in number at $-82^{\circ}$ in panel (d), some of which intersect the southern and some the northern magnetopause, the latter thus passing through the equatorial plane. At $-86^{\circ}$ and $-90^{\circ}$ in panels (e) and (f) all field lines are open, and intersect the southern magnetopause. Figure 8 shows the intersections of the open field lines with the magnetopause in a similar format to Fig. 4. Panels (a) and (b) show the intersections with the southern and northern magnetopause, respectively.

Turning now to the boundaries of the southern dayside UV oval shown by the red crosses in image A in Fig. 2, the corresponding model field lines are shown in Fig. 9 in the same format as Fig. 5. The field lines corresponding to the equatorward boundary shown by the blue lines in panel (a) are again all closed. In the dawn and post-noon sector they extend in the equatorial plane to $\sim 11 R_{\mathrm{S}}$ similar to case $\mathrm{C}$, while moving inwards to $\sim 8.5 R_{\mathrm{S}}$ in the pre-noon sector as the equatorward boundary in the image moves to somewhat lower latitudes. For the field lines corresponding to the poleward boundary shown in panel (b), we see from Fig. 2 that most of them lie somewhat poleward of the model open-closed field boundary and are thus open (black lines), mapping to the southern magnetopause. Only one of these model boundary field lines is closed, lying in the pre-noon sector, shown by the purple line in panel (b). This field line maps to a radial distance of $\sim 20.5 R_{\mathrm{S}}$ in the equatorial plane, close to the post-dawn magnetopause.

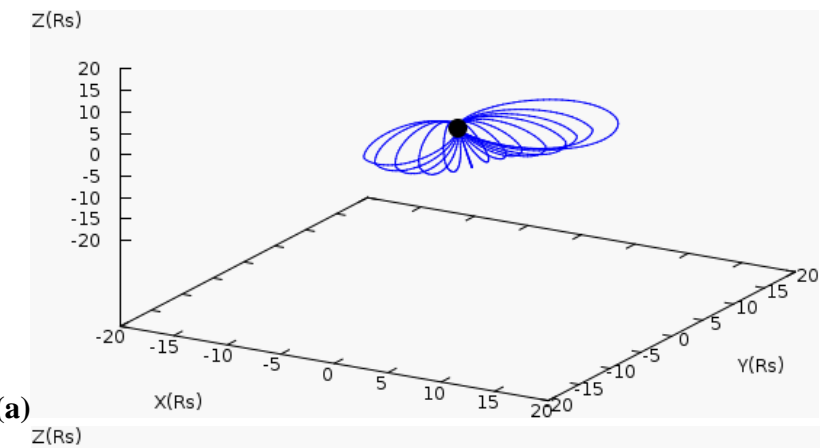

${ }^{\text {(a) }}$ (Rs)

(b)

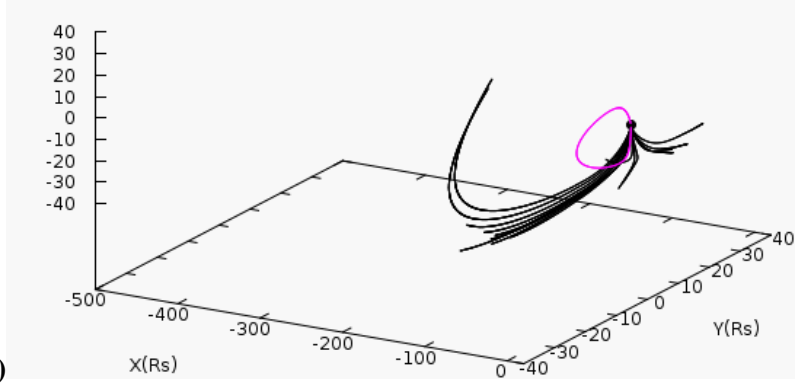

Fig. 9. Field lines mapping to (a) the equatorward and (b) the poleward boundary of the dayside UV auroral oval for image A with $k_{\mathrm{S}}=0.2$, corresponding in the southern ionosphere to the positions indicated by the red crosses in Fig. 2. The format is the same as for Fig. 5.

The equatorial intersections of these boundary field lines are depicted in Fig. 10 in a similar format to Fig. 6, where panel (a) uses the same spatial scales as Fig. 6 showing the near-planet region, while panel (b) shows a larger region extending down the magnetospheric tail. The closed field region is contained between the red and green lines, the former being projected from the southern ionosphere and the latter from the northern, which now extends to much greater distances down the tail than for case $\mathrm{C}$. These lines are connected to each other at intersections of the magnetic separator line with the equatorial plane. (For a discussion of the "open" field geometries occurring for northward- and southwarddirected IMF in these cases, corresponding to southward- and northward-directed IMF at Earth due to the differing planetary dipole directions, the reader is referred to the works of Alexeev and Belenkaya (1983) and Blomberg et al. (2005), and references therein.) The blue squares again show the equatorial intersections of the equatorward boundary of the dayside oval, which as for case $\mathrm{C}$ is somewhat variably located between the outer edge and centre of the ring current region, $\sim 8.5-12.5 R_{\mathrm{S}}$. In this case, however, it is located near the outer edge of the ring current in the dawn and postnoon sector, and near the centre in the pre-noon sector. With regard to the poleward boundary, only one of the model field lines intersects the equator, shown by the purple square close to the magnetopause in the post-dawn sector. 


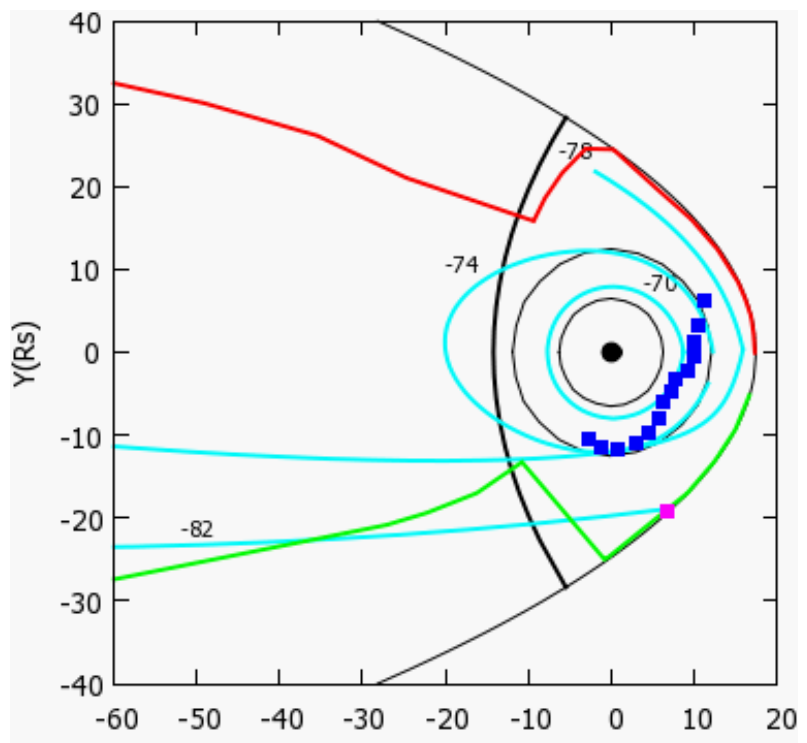

(a)

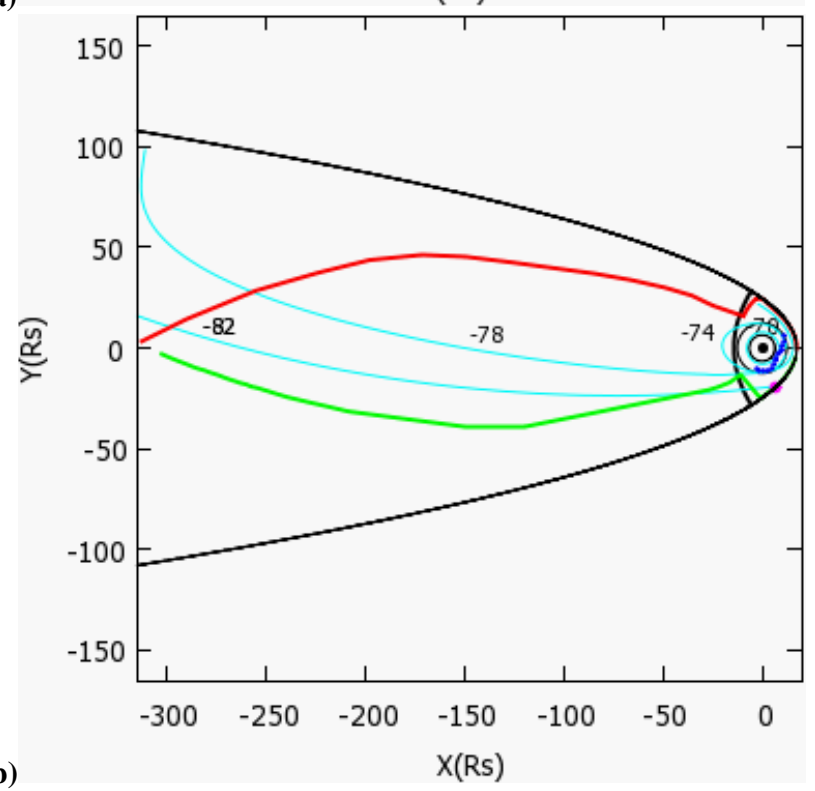

Fig. 10. Mappings of field lines into the KSM equatorial plane for image A with $k_{\mathrm{S}}=0.2$, in a format similar to Fig. 6. Panel (a) shows the near-planet region exactly as in Fig. 6, while panel (b) shows a larger region extending into the magnetospheric tail. The closed field region is contained between the red and green lines, where the former is projected from the Southern Hemisphere and the latter from the Northern.

Overall, however, despite the very different IMF conditions prevailing and the consequent significantly different outer magnetospheric model field geometries, as for image $\mathrm{C}$ these results imply that the dayside oval maps between the outer region of the ring current and the open-closed field boundary. We have again tested the generality of this result by comparison with one of the corresponding images in the January 2004 data set examined by Belenkaya et al. (2007), specifically image " $k$ " obtained under similarly compressed southward IMF conditions on 26 January 2004. Belenkaya et al. (2007) again showed that the poleward boundary of the aurora in this case matches well with the boundary of open field lines, while here we have newly shown that the mapping of the equatorward boundary in this image where it is clearly visible in the dawn to noon sector is very comparable to that shown here for case A in Fig. 10. Again, the former of these locations matches the considerations of Cowley et al. (2004a, b, 2008), and the latter those of Sittler et al. (2006).

We finally note with regard to these results that open field lines with distinctly differing characteristics can exist in Saturn's tail under southward IMF conditions, not only field lines that pass from the open region in the ionosphere directly to the magnetopause in the same hemisphere, but also open lines that pass through the equatorial plane from the ionosphere in one hemisphere to the magnetopause in the other. The layer lying on the magnetopause side of the red line in Fig. 10 contains such open field lines that map to the southern ionosphere, while the corresponding layer on the magnetopause side of the green line contains such open field lines that map to the northern ionosphere. It seems possible that these differing types of open field regions may then sustain differing flow regimes in the ionosphere. We note particularly that the results of Kane et al. (2008) and McAndrews et al. (2009) suggest sub-corotating flow throughout the equatorial nightside magnetosphere to radial distances of at least $\sim 50 R_{\mathrm{S}}$, while flows on field lines that do not intersect the equatorial region may be distinctly different.

\section{Summary, discussion, and conclusions}

In this paper we have examined the mapping of the dayside UV oval along field lines into Saturn's magnetosphere by combining auroral images obtained by the HST with a global model of the magnetospheric magnetic field. Previous results have shown that the UV oval maps to the outer parts of the magnetosphere near the open-closed field boundary. Since the field structure in this region depends significantly on the orientation of the IMF, as shown by earlier studies at Earth and Jupiter as well as Saturn, realistic field modelling requires simultaneous knowledge of the upstream IMF. To date only two such simultaneous data sets of this nature exist for Saturn, one obtained in January 2004 during Cassini approach at distances from the planet of $\sim 1300 R_{\mathrm{S}}$, resulting in significant IMF propagation time uncertainties, and a second in February 2008 when four UV image sequences were obtained when Cassini was in the solar wind just upstream from the dayside bow shock. Here we have focussed on the latter data, specifically on two of the four UV images showing the pre-equinox southern dayside oval, which exemplify conditions for northward and southward IMF. However, we have also compared results with those for two related images in the January 2004 data set. We emphasise, however, 
that the auroral distributions observed in these images are entirely representative of a much wider body of such data (e.g., Clarke et al., 2009).

Calculations of the field structure were thus undertaken using the paraboloid magnetosphere model appropriate to the conditions prevailing during these HST imaging intervals, corresponding in the February 2008 data to an interval of significant magnetospheric compression by the solar wind (Clarke et al., 2009; Belenkaya et al., 2010), with a penetrating field computed from suitably lagged and averaged Cassini IMF data. The auroral boundaries were then mapped along the model field lines in each case from the ionosphere to the magnetosphere, with the following principal results.

(a) Although the model field structure in the outer magnetosphere and tail is very different in the two cases, as expected from the differing IMF directions prevailing, the mapped dayside UV auroral oval is found nevertheless to have a consistent location relative to the field, spanning the outer magnetosphere between the outer part of the ring current and the open-closed field boundary at the magnetopause. Corresponding results have also been found for selected images within the January 2004 data set obtained under related interplanetary conditions, thus supporting the generality of these findings. The results are in excellent accord with the findings of Talboys et al. (2009), who studied Cassini observations of field-aligned current signatures in high-latitude dayside magnetic field data, and found that the region of upward currents spanned the outer magnetosphere from near the outer boundary of the ring current to near the boundary of open field lines. Upward currents are potentially associated with downward accelerated electrons and bright aurora. This outer magnetospheric region is that dominated by hot tenuous plasma, rather than by cool dense plasma characteristic of the inner region (Schippers et al., 2008; Sergis et al., 2009; Kellett et al., 2010, 2011).

(b) In more detail, the equatorward boundary of the dayside oval in images $\mathrm{A}$ and $\mathrm{C}$ was found to map typically to distances of $\sim 12 R_{\mathrm{S}}$ in the equatorial plane, near the outer boundary of the model ring current appropriate to the compressed magnetospheric conditions prevailing. However, this boundary was also found to map somewhat further inward to near the centre of the ring current at $\sim 9 R_{\mathrm{S}}$ in some restricted sectors, near dusk in image $\mathrm{C}$, and in the pre-noon sector in image A. Under the more typical less compressed conditions of image " $m$ " in the January 2004 data set, the equatorward boundary was found to map consistently to a radial distance of $\sim 15 R_{\mathrm{S}}$ in the equatorial plane, again close to the outer boundary of the ring current. The poleward boundary of the dayside oval, on the other hand, was found typically to straddle the boundary between open and closed field lines, in conformity with the previous conclusions of Belenkaya et al. $(2006 b, 2007,2008,2010)$. Oval boundary points on the closed field side thus map close to the dayside magnetopause.

(c) These results have implications with regard to previous theoretical considerations concerning the origins and mapping of the auroras. Specifically we note that the association of the equatorward boundary of the dayside oval with the outer edge of the ring current agrees with the discussion of Sittler et al. (2006), while the association of the poleward boundary of the oval with the open-closed field boundary relates to the discussion of Cowley et al. (2004a, $b, 2008$ ). We find that the UV oval does not map inward to the primary corotation breakdown region found by Wilson et al. (2009) at radial distances of $\sim 3-4 R_{\mathrm{S}}$ within the inner Enceladus torus. This maps to the southern ionosphere at a latitude of $-62^{\circ}$, well equatorward of the equatorward boundary of the UV oval at $\sim-70^{\circ}$ to $-74^{\circ}$, in agreement with the prior discussion of Cowley and Bunce (2003). However, the field-aligned currents associated with this inner corotation breakdown may well be associated with a lowerlatitude "secondary" oval observed in IR data by Stallard et al. $(2008,2010)$.

We finally note the very different field structures occurring in Saturn's outer magnetosphere and tail within the paraboloid models computed here for northward and southward-directed IMF, even for the rather small interplanetary field strengths prevailing. These may then give rise to differing IMF-dependent flow regimes on high-latitude open field lines, for example, between open field lines that pass directly from the ionosphere to the magnetopause in the corresponding hemisphere, and those that pass to the magnetopause in the opposite hemisphere through the equatorial plane, where they may be significantly influenced by magnetospheric plasma dynamics. For northward IMF only the former type of open field line is present, while for southward IMF both types are present in differing regions of the open polar cap. Further study of these features may be of interest.

Acknowledgements. Work at the Institute of Nuclear Physics (INP), Moscow State University, and the University of Leicester (UoL) was supported by RFBR (Grant No 09-02-92603-KO_a) and London Royal Society (JP080836) Joint Project funding. Work was also supported at the INP by RFBR Grants 09-05-00798 and 1105-00894, and at the UoL by STFC grant ST/H002480/1. ESB is thankful to EU FP7 projects EUROPLANET/JRA3 and IMPEX for support. The authors thank I. I. Alexeev (INP) for fruitful scientific discussions. This work employs observations made with the NASA/ESA Hubble Space Telescope, obtained at the Space Telescope Science Institute, which is operated by AURA Inc for NASA.

Topical Editor I. A. Daglis thanks three anonymous referees for their help in evaluating this paper.

\section{References}

Alexeev, I. I.: The magnetospheres of Mercury, Earth, and the giant planets Jupiter and Saturn, Moscow Univ. Phys. Bull., 65, 300306, 2010.

Alexeev, I. I. and Belenkaya, E. S.: Electric field in an open model of the magnetosphere, Geomagn. Aeron., 23, 57-61, 1983. 
Alexeev, I. I. and Belenkaya, E. S.: Modeling of the Jovian Magnetosphere, Ann. Geophys., 23, 809-826, doi:10.5194/angeo-23809-2005, 2005.

Alexeev, I. I., Kalegaev, V. V., Belenkaya, E. S., Bobrovnikov, S. Y., Bunce, E. J., Cowley, S. W. H., and Nichols, J. D.: A global magnetic model of Saturn's magnetosphere, and a comparison with Cassini SOI data, Geophys. Res. Lett., 33, L08101, doi:10.1029/2006GL025896, 2006.

Andrews, D. J., Cowley, S. W. H., Dougherty, M. K., and Provan, G.: Magnetic field oscillations near the planetary period in Saturn's equatorial magnetosphere: Variation of amplitude and phase with radial distance and local time, J. Geophys. Res., 115, A04212, doi:10.1029/2009JA014729, 2010.

Arridge, C. S., Russell, C. T., Khurana, K. K., Achilleos, N., Cowley, S. W. H., Dougherty, M. K., Southwood, D. J., and Bunce, E. J.: Saturn's magnetodisc current sheet, J. Geophys. Res., 113, A04214, doi:10.1029/2007JA012540, 2008.

Badman, S. V., Cowley, S. W. H., Gérard, J.-C., and Grodent, D.: A statistical analysis of the location and width of Saturn's southern auroras, Ann. Geophys., 24, 3533-3545, doi:10.5194/angeo-243533-2006, 2006.

Belenkaya, E. S., Alexeev, I. I., Kalegaev, V. V., and Blokhina, M. S.: Definition of Saturn's magnetospheric model parameters for the Pioneer 11 flyby, Ann. Geophys., 24, 1145-1156, doi:10.5194/angeo-24-1145-2006, 2006a.

Belenkaya, E. S., Cowley, S. W. H., and Alexeev, I. I.: Saturn's aurora in the January 2004 events, Ann. Geophys., 24, 16491663, doi:10.5194/angeo-24-1649-2006, 2006b.

Belenkaya, E. S., Alexeev, I. I., Blokhina, M. S., Cowley, S. W. H., Badman, S. V., Kalegaev, V. V., and Grigoryan, M. S.: IMF dependence of the open-closed field line boundary in Saturn's ionosphere, and its relation to the UV auroral oval observed by the Hubble Space Telescope, Ann. Geophys., 25, 1215-1226, doi:10.5194/angeo-25-1215-2007, 2007.

Belenkaya, E. S., Cowley, S. W. H., Badman, S. V., Blokhina, M. S., and Kalegaev, V. V.: Dependence of the open-closed field line boundary in Saturn's ionosphere on both the IMF and solar wind dynamic pressure: comparison with the UV auroral oval observed by the HST, Ann. Geophys., 26, 159-166, doi:10.5194/angeo-26-159-2008, 2008.

Belenkaya, E. S., Alexeev, I. I., Blokhina, M. S., Bunce, E. J., Cowley, S. W. H., Nichols, J. D., Kalegaev, V. V., Petrov, V. G., and Provan, G.: IMF dependence of Saturn's auroras: modelling study of HST and Cassini data from 12-15 February 2008, Ann. Geophys., 28, 1559-1570, doi:10.5194/angeo-281559-2010, 2010.

Blomberg, L. G., Cumnock, J. A., Alexeev, I. I., Belenkaya, E. S., Bobrovnikov, S. Yu., and Kalegaev, V. V.: Transpolar aurora: time evolution, associated convection patterns, and a possible cause, Ann. Geophys., 23, 1917-1930, doi:10.5194/angeo-231917-2005, 2005.

Bunce, E. J., Cowley, S. W. H., Alexeev, I. I., Arridge, C. S., Dougherty, M. K., Nichols, J. D., and Russell, C. T.: Cassini observations of the variation of Saturn's ring current parameters with system size, J. Geophys. Res., 112, A10202, doi:10.1029/2007JA012275, 2007

Bunce, E. J., Arridge, C. S., Clarke, J. T., Coates, A. J., Cowley, S. W. H., Dougherty, M. K., Gérard, J.-C., Grodent, D., Hansen, K. C., Nichols, J. D., Southwood, D. J., and Tal- boys, D. L.: Origin of Saturn's aurora: Simultaneous observations by Cassini and the Hubble Space Telescope, J. Geophys. Res., 113, A09209, doi:10.1029/2008JA013257, 2008a.

Bunce, E. J., Arridge, C. S., Cowley, S. W. H., and Dougherty, M. K.: Magnetic field structure of Saturn's dayside magnetosphere and its mapping to the ionosphere: Results from ring-current modelling, J. Geophys. Res., 113, A02207, doi:10.1029/2007JA012538, 2008b.

Clarke, J. T., Nichols, J., Gérard, J.-C., Grodent, D., Hansen, K. C., Kurth, W., Gladstone, G. R., Duval, J., Wannawichian, S., Bunce, E. J., Cowley, S. W. H., Crary, F., Dougherty, M., Lamy, L., Mitchell, D., Pryor, W., Retherford, K., Stallard, T., Zieger, B., Zarka, P., and Cecconi, B.: Response of Jupiter's and Saturn's auroral activity to the solar wind, J. Geophys. Res., 114, A05210, doi:10.1029/2008JA013694, 2009.

Cowley, S. W. H. and Bunce, E. J.: Corotation-driven magnetosphere-ionosphere coupling currents in Saturns magnetosphere and their relation to the auroras, Ann. Geophys., 21, 1691-1707, doi:10.5194/angeo-21-1691-2003, 2003.

Cowley, S. W. H. and Hughes, W. J.: Observation of an IMF sector effect in the Y magnetic field component at geostationary orbit, Planet. Space Sci., 31, 73-90, 1983.

Cowley, S. W. H., Bunce, E. J., and O'Rourke, J. M.: A simple quantitative model of plasma flows and currents in Saturn's polar ionosphere, J. Geophys. Res., 109, A05212, doi:10.1029/2003JA010375, 2004a.

Cowley, S. W. H., Bunce, E. J., and Prangé, R.: Saturn's polar ionospheric flows and their relation to the main auroral oval, Ann. Geophys., 22, 1379-1394, doi:10.5194/angeo-22-13792004, 2004b.

Cowley, S. W. H., Arridge, C. S., Bunce, E. J., Clarke, J. T., Coates, A. J., Dougherty, M. K., Gérard, J.-C., Grodent, D., Nichols, J. D., and Talboys, D. L.: Auroral current systems in Saturn's magnetosphere: comparison of theoretical models with Cassini and HST observations, Ann. Geophys., 26, 2613-2630, doi:10.5194/angeo-26-2613-2008, 2008.

Dougherty, M. K., Kellock, S., Southwood, D. J., Balogh, A., Smith, E. J., Tsurutani, B. T., Gerlach, B., Glassmeier, K.-H., Gleim, F., Russell, C. T., Erdos, G., Neubauer, F. M., and Cowley, S. W. H.: The Cassini magnetic field investigation, Space Sci. Rev., 114, 331-383, 2004.

Hill, T. W.: Inertial limit on corotation, J. Geophys. Res., 84, 6554 6558, 1979.

Jackman, C. M., Arridge, C. S., Krupp, N., Bunce, E. J., Mitchell, D. G., Kurth, W. S., McAndrews, H. J., Dougherty, M. K., Russell, C. T., Achilleos, N., Coates, A. J., and Jones, G. H.: A multi-instrument view of tail reconnection at Saturn, J. Geophys. Res., 113, A11213, doi:10.1029/2008JA013592, 2008.

Kanani, S. J., Arridge, C. S., Jones, G. H., Fazakerley, A. N., McAndrews, H. J., Sergis, N., Krimigis, S. M., Dougherty, M. K., Coates, A. J., Young, D. T., Hansen, K. C., and Krupp, N.: A new form of Saturn's magnetopause using a dynamic pressure balance model, based on in situ, multi-instrument Cassini measurements, J. Geophys. Res., 115, A06207, doi:10.1029/2009JA014262, 2010.

Kane, M., Mitchell, D. G., Carbary, J. F., Krimigis, S. M., and Crary, F. J.: Plasma convection in Saturn's outer magnetosphere determined from ions detected by the Cassini INCA experiment, 
Geophys. Res. Lett., 35, L04102, doi:10.1029/2007GL032342, 2008.

Kellett, S., Bunce, E. J., Coates, A. J., and Cowley, S. W. H.: Thickness of Saturn's ring current determined from north-south Cassini passes through the current layer, J. Geophys. Res., 114, A04209, doi:10.1029/2008JA013942, 2009.

Kellett, S., Arridge, C. S., Bunce, E. J., Coates, A. J., Cowley, S. W. H., Dougherty, M. K., Persoon, A. M., Sergis, N., and Wilson, R. J.: Nature of the ring current in Saturn's dayside magnetosphere, J. Geophys. Res., 115, A08201, doi:10.1029/2009JA015146, 2010.

Kellett, S., Arridge, C. S., Bunce, E. J., Coates, A. J., Cowley, S. W. H., Dougherty, M. K., Persoon, A. M., Sergis, N., and Wilson, R. J.: Saturn's ring current: Local time dependence and temporal variability, J. Geophys. Res., 116, A05220, doi:10.1029/2010JA016216, 2011.

Kurth, W. S., Bunce, E. J., Clarke, J. T., Crary, F. J., Grodent, D. G., Ingersoll, A. P., Dyudina, U. A., Lamy, L., Mitchell, D. G., Persoon, A. M., Pryor, W. R., Saur, J., and Stallard, T.: Auroral processes, in: Saturn from Cassini-Huygens, edited by: Dougherty, M. K., Esposito, L. W., and Krimigis, S. M., Springer Science \& Business Media, pp. 333-374, doi:10.1007/978-14020-9217-6_12, 2009.

McAndrews, H. J., Thomsen, M. F., Arridge, C. S., Jackman, C. M., Wilson, R. J., Henderson, M. G., Tokar, R. L., Khurana, K. K., Sittler, E. C., Coates, A. J., and Dougherty, M. K.: Plasma in Saturn's nightside magnetosphere and the implications for global circulation, Planet. Space Sci., 57, 1714-1722, 2009.

Milan, S. E., Cowley, S. W. H., Lester, M., Wright, D. M., Slavin, J. A., Fillingim, M., Carlson, C. W., and Singer, H. J.: Response of the magnetotail to changes in the open flux content of the magnetosphere, J. Geophys. Res., 109, A04220, doi:10.1029/2003JA010350, 2004.

Nichols, J. D., Cowley, S. W. H., and Lamy, L.: Dawn-dusk oscillation of Saturn's conjugate auroral ovals, Geophys. Res. Lett., 37, L24102, doi:10.1029/2010GL045818, 2010.

Pontius, D. H. and Hill, T.W.: Departure from corotation of the Io plasma torus: local plasma production, Geophys. Res. Lett., 9, 1321-1324, 1982.

Pontius Jr., D. H. and Hill, T. W.: Plasma mass loading from the extended neutral gas torus of Enceladus as inferred from the observed corotation lag, Geophys. Res. Lett., 36, L23103, doi:10.1029/2009GL041030, 2009.

Saur, J., Mauk, B. H., Kassner, A., and Neubauer, F. M.: A model for the azimuthal plasma velocity in Saturn's magnetosphere, J. Geophys. Res., 109, A05217, doi:10.1029/2003JA010207, 2004.
Schippers, P., Blanc, M., André, N., Dandouras, I., Lewis, G. R., Gilbert, L. K., Persoon, A. M., Krupp, N., Gurnett, D. A., Coates, A. J., Krimigis, S. M., Young, D. T., and Dougherty, M. K.: Multi-instrument analysis of electron populations in Saturn's magnetosphere, J. Geophys. Res., 113, A07208, doi:10.1029/2008JA013098, 2008.

Sergis, N., Krimigis, S. M., Mitchell, D. G., Hamilton, D. C., Krupp, N., Mauk, E. C., Roelof, E. C., and Dougherty, M. K.: Energetic particle pressure in Saturn's magnetosphere measured with the Magnetospheric Imaging Instrument on Cassini, J. Geophys. Res., 114, A02214, doi:10.1029/2008JA013774, 2009.

Sittler, E. C., Blanc, M. F., and Richardson, J. D.: Proposed model for Saturn's auroral response to the solar wind: Centrifugal instability model, J. Geophys. Res., 111, A06208, doi:10.1029/2005JA011191, 2006.

Stallard, T., Miller, S., Melin, H, Lystrup, M., Dougherty, M., and Achilleos, N.: Saturn's auroral/polar $\mathrm{H}_{3}^{+}$infrared emission: I. General morphology and ion velocity structure, Icarus, 189, 113, 2007a.

Stallard, T., Smith, C., Miller, S., Melin, H., Lystrup, M., Aylward, A., and Dougherty, M.: Saturn's auroral/polar $\mathrm{H}_{3}^{+}$infrared emission: II. A comparison with plasma flow models, Icarus, 191, 678-690, 2007b.

Stallard, T. S., Miller, S., Melin, H., Lystrup, M., Cowley, S. W. H., Bunce, E. J., Achilleos, N., and Dougherty, M.: Jovian-like aurorae on Saturn, Nature, 453, 1083-1085, 2008.

Stallard, T., Melin, H., Cowley, S. W. H., Miller, S., and Lystrup, M. P., Location and magnetospheric mapping of Saturn's mid-latitude infrared auroral oval, Ap. J. Lett., 722, L85L89, 2010.

Talboys, D. L., Arridge, C. S., Bunce, E. J., Coates, A. J., Cowley, S. W. H., and Dougherty, M. K.: Characterization of auroral current systems in Saturn's magnetosphere: Highlatitude Cassini observations, J. Geophys. Res., 114, A06220, doi:10.1029/2008JA013846, 2009.

Tsurutani, B. T., Jones, D. E., Lepping, R. P., Smith, E. J., and Sibeck, D. G.: The relationship between the IMF $B_{y}$ and the distant tail (150-238 $\mathrm{R}_{e}$ ) lobe and plasma sheet $\mathrm{B}_{y}$ fields, Geophys. Res. Lett., 11, 1082-1085, 1984.

Vasyliunas, V. M.: Plasma distribution and flow, in: Physics of the Jovian Magnetosphere, edited by: Dessler, A. J., p. 395, Cambridge Univ. Press, Cambridge, UK, 1983.

Wilson, R. J., Tokar, R. L., and Henderson, M. G.: Thermal ion flow in Saturn's inner magnetosphere measured by the Cassini plasma spectrometer: A signature of the Enceladus torus?, Geophys. Res. Lett., 36, L23104, doi:10.1029/2009GL040225, 2009. 